\title{
Une carte et une base de données pour les formations à silex du sud de la France : un outil pour la pétroarchéologie
}

A map and a database for flint bearing formations in Southern France: a tool for

Petroarchaeology

Paul Fernandes, Jean-Paul Raynal, Pascal Tallet, Christophe Tuffery, Michel Piboule, Micheline Séronie-Vivien, Marie-Roger Séronie-Vivien, Alain Turq, André Morala, Jehanne Affolter, Dominique Millet, Françoise Millet, Fréderic Bazile, Patrick Schmidt, Pascal Foucher, Vincent Delvigne, Jérémie Liagre, Stéphane Gaillot, Alexandre Morin, Marie-Hélène Moncel, Jean-François Garnier et Céline Léandri-Bressy

\section{OpenEdition}

\section{Journals}

Édition électronique

URL : http://journals.openedition.org/paleo/2633

DOI : $10.4000 /$ paleo.2633

ISSN : $2101-0420$

Éditeur

SAMRA

Édition imprimée

Date de publication : 15 décembre 2013

Pagination : $219-228$

ISSN : $1145-3370$

Référence électronique

Paul Fernandes, Jean-Paul Raynal, Pascal Tallet, Christophe Tuffery, Michel Piboule, Micheline Séronie-Vivien, Marie-Roger Séronie-Vivien, Alain Turq, André Morala, Jehanne Affolter, Dominique Millet, Françoise Millet, Fréderic Bazile, Patrick Schmidt, Pascal Foucher, Vincent Delvigne, Jérémie Liagre, Stéphane Gaillot, Alexandre Morin, Marie-Hélène Moncel, Jean-François Garnier et Céline Léandri-Bressy, " Une carte et une base de données pour les formations à silex du sud de la France : un outil pour la pétroarchéologie », PALEO [En ligne], 24 | 2013, mis en ligne le 17 avril 2014, consulté le 07 juillet 2020. URL : http://journals.openedition.org/paleo/2633; DOI : https://doi.org/10.4000/paleo. 2633

Ce document a été généré automatiquement le 7 juillet 2020

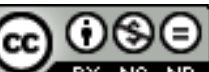

PALEO est mis à disposition selon les termes de la licence Creative Commons Attribution - Pas d'Utilisation Commerciale - Pas de Modification 4.0 International. 


\section{Une carte et une base de données pour les formations à silex du sud de la France : un outil pour la pétroarchéologie}

A map and a database for flint bearing formations in Southern France: a tool for Petroarchaeology

Paul Fernandes, Jean-Paul Raynal, Pascal Tallet, Christophe Tuffery, Michel Piboule, Micheline Séronie-Vivien, Marie-Roger Séronie-Vivien, Alain Turq, André Morala, Jehanne Affolter, Dominique Millet, Françoise Millet, Fréderic Bazile, Patrick Schmidt, Pascal Foucher, Vincent Delvigne, Jérémie Liagre, Stéphane Gaillot, Alexandre Morin, Marie-Hélène Moncel, Jean-François Garnier et Céline Léandri-Bressy

\section{Introduction}

La nécessité de déterminer la provenance de la matière première ayant servi à la fabrication des outils est aussi ancienne que les premières recherches des préhistoriens (Damour 1865). Ce type de démarche est souvent biaisé du fait d'une méconnaissance du domaine minéral réellement exploité par les hommes préhistoriques. Des difficultés existent également pour constituer, harmoniser, échanger et diffuser des bases de données utilisables. Cet article présente un exemple de démarche suprarégionale et pluridisciplinaire qui a pour but d'élaborer un outil fiable au service de la reconstitution des géo-domaines d'approvisionnement.

\section{Des difficultés méthodologiques persistantes}

2 La pétroarchéologie est une discipline qui se pratique généralement à l'échelle locale, voire régionale. Sa pertinence repose sur la connaissance des disponibilités en matières 
premières régionales et plus rarement extrarégionales. Les limites administratives sont souvent inadéquates face aux questionnements archéologiques relatifs à la circulation des hommes et des matériaux. Le degré d'exhaustivité des collections de référence est très inégal d'une région à l'autre. Le manque de contact entre prospecteurs est patent, conduisant à un cloisonnement de la discipline, avec les dérives des problématiques et du vocabulaire utilisé qui en découlent. Les données ne sont généralement pas comparables, car obtenues par des protocoles d'acquisition différents.

3 À défaut d'une véritable démarche unitaire appelée par certains (Turq 2005), un renforcement des collaborations entre les différents acteurs de la pétroarchéologie est indispensable pour aboutir à la mise en service de véritables plateformes d'informations. La prospection des gites n'est jamais exhaustive et nous ne souscrivons pas à la démarche de Primault (2003) qui donne une représentation des gîtes inventoriés sur une aire géographique limitée. En outre, la diversité géologique des formations à silex implique de détailler, pour chaque affleurement, le contexte géologique. Regrouper cette information avec le descriptif des silex collectés est nécessaire pour la démarche pétroarchéologique car elle permet d'atteindre une meilleure connaissance du matériau.

\section{L'origine du projet}

4 Les premières cartes furent élaborées d'après un protocole mis au point en 2007 (Bressy et al. 2007) à partir des données collectées lors des prospections des membres du PCR "réseau de Lithothèques en Rhône-Alpes": Affolter (Bauges, Bornes, Bugey, Jura, Préalpes médianes, Savoie, Vercors); Morin et Cousseran (vallée du Buech); Bressy (Bauges, Buëch, Bugey, Chartreuse, Chambaran, Diois, vallée de l'Isère, Royans, Vercors nord); Féblots-Augustin (Bugey, Ain); le CAP Valence, Beeching et Brochier (pays de Marsanne et de Valdaine, Tricastin, vallée du Rhône, sud Baronnies); Bintz (Chartreuse, Diois, Isère,Vercors) ; Grünwald (Vercors, Royans, Isère, Diois, Chartreuse, Bugey); Fernandes (Auvergne, Cruas, Rochemaure, Saint-Montant, Saint-Marceld'Ardèche, Barjac-Issirac, Laval-Saint-Roman, Carsan, Vans, Aubenas, Crest,Taulignan) ; Masson (Jura, Savoie) ; Picavet (Vercors sud) ; Piboule (Auvergne, Loire, Cruas, BarjacIssirac, Laval-Saint-Roman); Raynal (Haute-Loire), Riche (Vassieux); Vilain (Buggey, Savoie). Ces données géoréférencées utilisaient des systèmes de coordonnées différents (Lambert 2 zone, Lambert 2 étendu, WGS 84) qu'il a fallu homogénéiser.

5 Une fois harmonisées, ces données ont servi à visualiser les points de prélèvements dans les régions Rhône-Alpes et Auvergne. Les premiers essais furent réalisés dans le cadre des prospections thématiques en Auvergne et Languedoc-Roussillon (Lozère) débutées en 2003 et étendues à l'Ardèche, en lien avec l'étude de l'origine des silex utilisés dans les sites de Saint-Anne I, Baume Vallée et Payre. L'intérêt des résultats acquis nous a convaincu qu'une comparaison plus ample des séries archéologiques entre elles permet d'enrichir les observations faites isolément, sur chaque site. Pour le Paléolithique moyen ancien, cette démarche permet de poser la question des relations entre vallée du Rhône et Massif central. La qualité et l'importance de la documentation acquise devenaient suffisantes pour établir un modèle qui dépasse les limites géographiques régionales.

6 Les cartes obtenues lors de cette étape permettaient de visualiser la localisation topographique, l'origine stratigraphique et le positionnement secondaire des gîtes. 
Durant les trois premières années, ce SIG a été essentiellement consacré à l'archivage d'informations géologiques, mais c'était insuffisant.

7 Ces représentations sous forme de points, bien que fidèles, ne traduisaient que partiellement l'étendue des formations sur lesquelles se trouvent les ressources minérales et encore moins la complexité de leurs aires de dispersion (ou de répartition). En outre, l'échelle régionale, si couramment utilisée, était inadaptée au niveau de précision recherché.

Dès 2009, nous nous sommes donc concentrés sur un mode de géoréférencement basé sur la représentation des formations. Cette nouvelle démarche a permis de fusionner les données de plusieurs cartes (Guibert 2000 ; Affolter 2009; Affolter et Bressy 2009; Bressy 2009...) ce qui n'était pas possible avec une représentation par gîte.

En 2011, nous avons décidé d'élargir le champ de nos investigations à tout le sud de la France afin de répondre aux problèmes posés par la présence de silex d'origine lointaine (plus de $100 \mathrm{~km}$ ) dans les séries archéologiques. La nouvelle carte établie indique les limites topographiques et l'origine stratigraphique des principales formations à silex dans six régions (Aquitaine, Auvergne, Languedoc-Roussillon, MidiPyrénées, Provence-Alpes - Côte-d'Azur, Rhône-Alpes) (fig. 1).

Figure 1 - carte des formations à silex du sud de la France. Echelle 1/3000000.

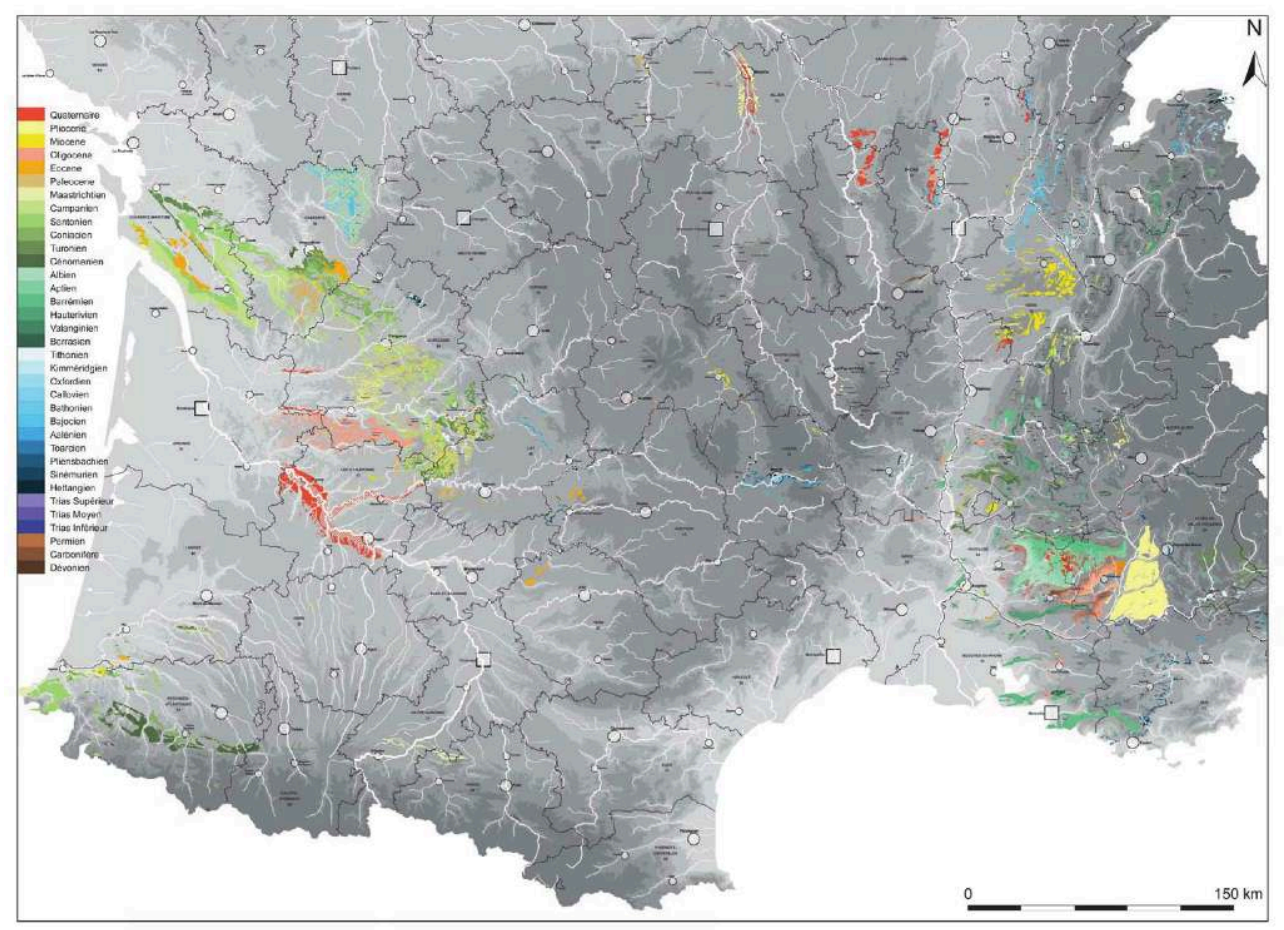

Nos connaissances, nos possibilités d'accès aux données et le réseau mis en place ne permettent pas de traiter, pour le moment, la totalité du territoire national. Notre carte est délimitée au nord par une ligne droite reliant La Rochelle à Bourg-en-Bresse. Ce découpage correspond aux secteurs sur lesquels nous avons accumulé un nombre suffisant de données de terrain. La carte n'est pas exhaustive : un tiers de la superficie retenue reste à compléter, ce qui ne pourra être réalisé qu'avec la participation de nouveaux collaborateurs. 
11 Le système de coordonnées cartographiques retenu est le Lambert 2 étendu. Ce système a été privilégié pour les raisons suivantes :

- validité du système pour l'ensemble de la France métropolitaine ;

- possibilité dans ArcGIS 9.2 de projeter les coordonnées de toutes origines dans ce système ;

- superposition possible dans ArcGIS 9.2 des données transformées avec les fichiers de la BD ALTIÒ à $50 \mathrm{~m}$ de l'IGN ;

- superposition possible dans ArcGIS 9.2 des données projetées avec les cartes géologiques scannées du BRGM à $1 / 50000$;

- affichage possible directement dans ArcGIS Explorer 900 des données pour un affichage en 3D/2D.

\section{Conception d'une carte renouvelée}

12 Cette seconde génération de cartes est le fruit d'une collaboration à l'échelle interrégionale entre des acteurs, pour la plupart impliqués dans la problématique relative à la provenance des silex archéologiques (Jean-Paul Raynal, Roger Séronie-Vivien, Michel Piboule, Alain Turq, André Morala, Jehanne Affolter, Pascal Foucher, Fréderic Bazile, Dominique et Françoise Millet, Vincent Delvigne, Marie-Hélène Moncel, René Liabeuf, Céline Bressy, Jean-Pierre Platel). Elle est le résultat d'un long travail collégial de prospections systématiques ou ciblées (en Auvergne, Rhône-Alpes, Provence Alpes Côte-d'Azur, Aquitaine, Poitou-Charentes et Midi-Pyrénées) et du dépouillement d'un grand nombre de documents. Hormis les informations directement collectées auprès de nos collaborateurs, le programme intègre des articles, thèses, monographies, rapports de PT et PCR, corrélés à l'analyse de 529 cartes géologiques à 1/50 000 et leurs notices, et de 252 fiches dossier BSS Info Terre qui offrent un accès aux descriptifs des forages et aux logs vérifiés.

13 Le protocole de recherche des formations à silex est le même pour chaque carte géologique. En premier lieu, sont replacés les gîtes déjà étudiés ou connus. Les recherches de nos prédécesseurs sont prises en compte: des cartes de formations à silex sont déjà disponibles et, plus souvent, des cartes de gîtes géoréférencés, symbolisés par des points. Ces lieux de collectes sont donc reportés sur les cartes géologiques afin de redessiner précisément les contours des formations contenant la matière première. En deuxième lieu, chaque notice est étudiée en cherchant les formations qui ont été décrites par les auteurs comme contenant des silex; en complément, les légendes dynamiques du site Info Terre sont également toutes passées en revue car le travail d'harmonisation entamé par le BRGM pour la vectorisation de ses cartes géologiques donne des informations supplémentaires.

14 Nous avons opté pour une représentation détaillée des formations, à l'échelle des cartes géologiques à $1 / 50000$. Ainsi, la qualité des données géologiques rassemblées est conservée sans perte ni simplification.

Sur ces nouvelles cartes figurent uniquement des polygones visualisant les affleurements primaires et des formations en position secondaire sans connexion avec la formation primaire. Ces formations sont distinguées en fonction de leur milieu de sédimentation ainsi que de leur situation stratigraphique et géomorphologique. Dans un premier temps, leur représentation est un PDF dynamique. 
Le déplacement de la souris sur une des formations recensées permet d'ouvrir une notice descriptive et explicative qui, lorsque la base de données sera complète, contiendra des photos aux trois échelles afin d'obtenir une fiche d'identité fiable de chaque faciès. La carte est suffisamment précise pour pouvoir « zoomer » à différentes échelles, du 1/1000000 au 1/25000. Les versions papier, à différentes échelles, permettent de visualiser aussi bien le potentiel minéral du sud de la France que celui d'un secteur particulier comme par exemple celui d'Audignon (Landes) (fig. 2).

Figure 2 - Carte des formations à silex de Chalosse et de l'Ouest-pyrénéen. Echelle 1/500000.

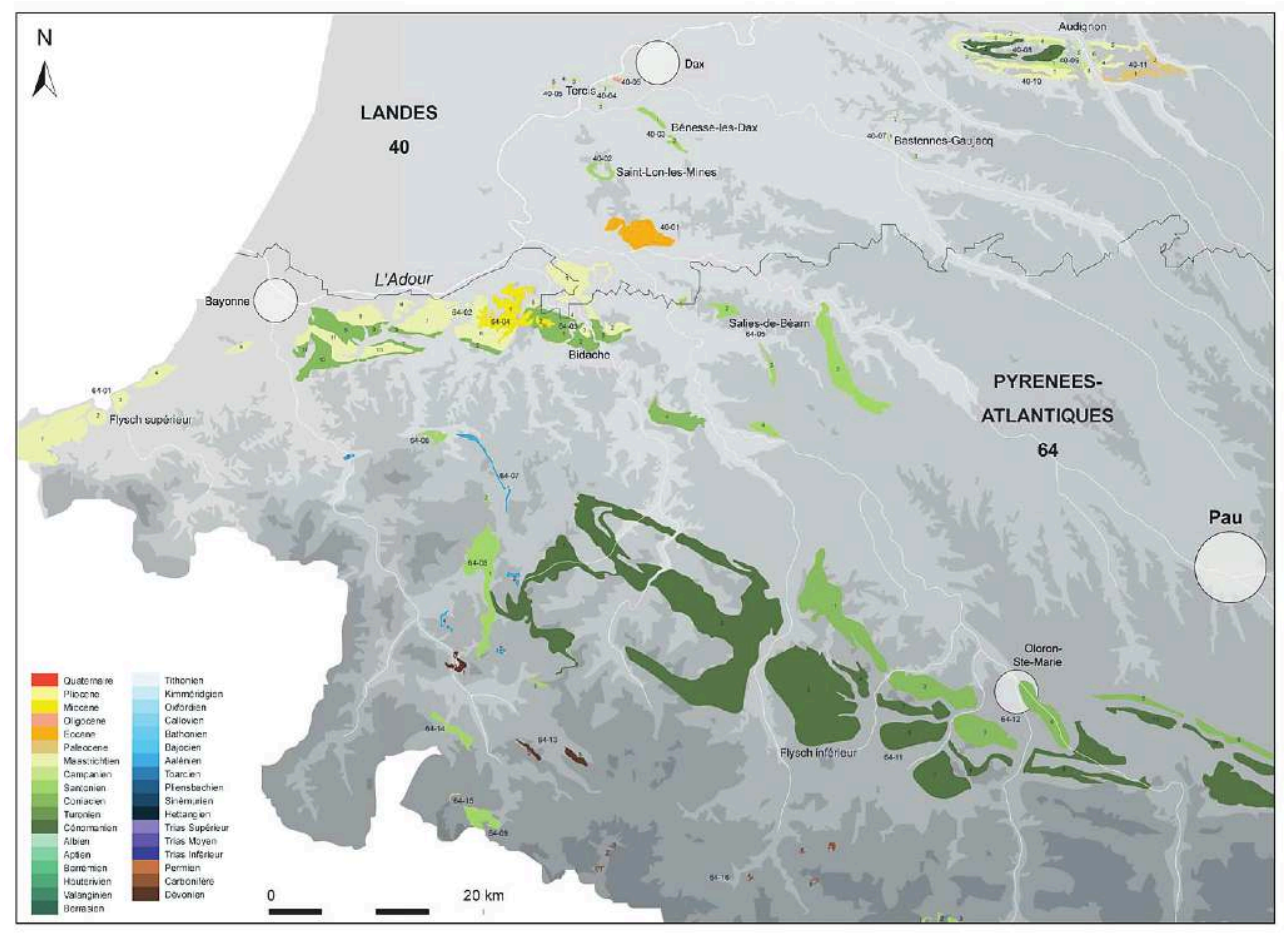

17 Le nom des formations doit avoir une valeur descriptive exprimée par un terme qui localise de façon précise l'entité géologique dénommée. Il fait généralement référence au toponyme le plus proche présent sur la carte IGN au 1/25 000. Pour les formations de grande extension, on retient le toponyme officiel de l'entité administrative la plus importante à l'intérieur de la zone ainsi circonscrite. Dans des cas exceptionnels et pour faciliter la compréhension, le terme choisi doit prendre en compte les noms déjà usités et publiés par les archéologues (dans des articles et les monographies) ou les géologues (dans les notices des cartes) : par exemple le Bergeracois qui désigne les silex issus du Campanien final, présents dans les altérites autour de Bergerac.

La dénomination géologique des formations est fidèle à la charte stratigraphique internationale ICS G. (Ogg 2010). Les couleurs des unités respectent en partie le programme de la carte géologique de la France au 1/50 000 (2003). Il a été abordé le problème de la variabilité au sein d'un même étage. C'est une trame qui distingue les variantes, l'étage conservant sa couleur standard.

La numérotation est simplifiée. Ce ne sont plus les points de collecte qui sont recensés mais l'ensemble de la formation porteuse du ou des même(s) type(s) de silex. « Le terme de formation désigne alors un terrain possédant des caractères communs et constituant un ensemble que l'on juge utile de distinguer » (Foucault et Raoult 1997). 

l'évolution des reliefs, ce qui implique une étude géomorphologique pour chaque gîte secondaire étudié. Notre expérience montre que la prise en compte de l'évolution du réseau hydrographique et des bassins versants est essentielle. Les matériaux présents dans ce type de formation fluviatile témoignent des polarités dynamiques (types d'itinéraires parcourus par les silex) dans la distribution entre les familles génétiques (silex collectés en position primaire) et les variantes gitologiques (issues de l'évolution des types génétiques). La reconstitution des déplacements naturels de chaque silex devient un élément essentiel de la pétroarchéologie. une matière première exploitable, soit en raison de l'inaccessibilité des rognons, soit en raison de la taille des éléments (trop petits), ou des cassures qui les affectent. La carte est, pour l'instant, une simple représentation du domaine minéral potentiel et non des ressources en silex exploitées. compilées et harmonisées dans une base de données ouverte. Actuellement, ces données sont en cours de géoréférencement.

\section{Inventaire et base iconographique accompagnant la représentation cartographique}

25 Chacune des formations recensées est donc accompagnée d'une notice qui décrit l'encaissant des accidents siliceux et le ou les type(s) de silex présent(s). Ces notices rappellent le nom et les travaux des inventeurs et des personnes qui ont travaillé à la caractérisation des silex. Elles sont rédigées par un des auteurs et revues par les autres membres du groupe de travail.

Nous donnons ici l'exemple de la fiche établie pour les formations de l'anticlinal d'Audignon (formations $\mathrm{n}^{\circ}$ 40-08, 40-09, 40-10, 40-11, fig. 2). Cette ride est l'une des plus étendues d'Aquitaine. Son axe est sensiblement parallèle à celui des Pyrénées. Elle s'étend sur une longueur de $28 \mathrm{~km}$ depuis Mugron à l'est jusqu'à Aire-sur-Adour à l'ouest. Les affleurements sont rares car la couverture mio-pliocène masque une partie du Crétacé. Le centre de l'anticlinal est formé de dépôts marins appartenant à l'Albien, au Cénomanien et au Turonien. Certains de ces étages contiennent des silex. Le Sénonien affleure sur les bordures. Le Maastrichtien est surtout présent au sud et sporadiquement sur le flanc nord. Cet ensemble est considéré comme la source d'un marqueur lithologique unique, le "silex de Chalosse à lépidorbitoïdes ». Il a été une très importante source de matières premières lithiques pour tous les hommes 
préhistoriques du Paléolithique ancien au Néolithique. Les dépôts de remaniement, riches en silex, sont concentrés sur les flancs de la structure et sont donc issus essentiellement des calcaires campaniens et maastrichtiens. L'observation des silex du Campanien n'a pas encore été réalisée.

\section{Détails de la fiche (Fernandes, Séronie-Vivien, Tallet) :}

-40-08 1-Audignon, 2-Brocas - c1-2 Cénomanien. Couches de Pilo: calcaires plus ou moins dolomitiques, blancs à beiges, contenant parfois accidents siliceux.

-40-09 1-Bidaou-Lasserre (Audignon), 2-Labay (Hauriet), 3-Becquerettes (Montaut), 4Haouriet (Banos, Audignon), 5-falaises de Daourat, 6-falaises de Berdoulon (Eyres-Moncube) - c6 Campanien. Couches de Pré-Marie : calcaires blancs à silex. Le Campanien débute par un horizon glauconieux puis le calcaire s'enrichit ensuite en silifications disposées en bancs parallèles à la stratification. La faune contient échinodermes, lamellibranches, nodosariidés et pithonelles. Les silex montrent une forte concentration en spicules uniaxes. Au sommet apparaissent en petits nombres des foraminifères de type orbitoïdes préfigurant les couches maastrichtiennes.

- 40-10 1-Cazaoubidaou-Barrère-Dumes (Montaut, Doazit, Horsarrieu, Dumes), 2-HaurietMaisonnave-Larrivière (Hauriet, Montaut, Banos), 3-Piréou, 4-Hillon, 5-Pirette (EyresMoncube, Montsoué) - c7 Maastrichtien. Couches de Dumes : calcaires beiges micritiques à rudistes, foraminifères, géodes de silice, silex bruns.

- 40-11 1- la Feuilleraie (Sarraziet), 2-rive gauche du Bahus (Bahus-Juzanx) - e1 Danien (sur la carte géologique). Les couches d'Arcet affleurent sur tout le pourtour du synclinal d'Audignon. Le niveau sommital, uniquement préservé à l'est, est constitué d'un calcaire récifal à polypiers, algues et échinides pouvant présenter des passées silicifiées. Les associations fauniques semblent indiquer un âge Paléocène supérieur (e2 Sélandien?) légèrement plus tardif que sur la carte.

Pour le Campanien, la coupe stratigraphique de référence se trouve près du village d'Eyre-Moncube, à l'est d'Audignon. C'est au toit de cette formation qu'apparaissent les silicifications. Il s'agit de bancs décimétriques et parallèles à la stratification. Ces silex contiennent des spicules, des fragments d'échinodermes, des lamellibranches et, comme dans les autres formations campaniennes de cette zone, des pithonelles. On note la présence d'un grand nombre de grains de glauconie dispersés dans une matrice le plus souvent microcristalline.

Nous avons collecté, en surface, au lieu-dit Barrère (Horsarrieu), des silex correspondant à cette description. Il s'agit de rognons réguliers à néo-cortex érodé. La couleur originelle est grise, ils deviennent blanchâtres et même blonds au cours de leur altération en position secondaire. Leur structure est homogène, leur texture est de type mudstone à wackestone. La matrice est microcristalline. Les formes peloïdes sont rares et ne dépassent pas les $20 \mu \mathrm{m}$. Les intraclastes sont peu nombreux (moins de $10 \%$ ). Ils sont de forme anguleuse et leur classement est bon. La macrofaune comprend des bivalves, des fragments d'échinodermes et même des ostracodes. Les spicules sont fréquents et les fragments de bryozoaires très rares. On observe surtout une faune planctonique (radiolaires, Globotruncana, valvulinidés); les formes benthiques sont très rares, nous n'avons pas observé de lépidorbitoides. De nombreux grains arrondis de glauconie dépassant parfois les $100 \mu \mathrm{m}$ sont dispersés dans la matrice. Dans la même formation colluvionnaire, on observe des silex beaucoup plus riches en formes 
benthiques (lépidorbitoïdes, orbitoïdes, Lagena) et qui appartiennent sans nul doute au Maastrichtien.

29 Les calcaires beiges maastrichtiens à rudistes de Dumes, Cazaoubidaou ou de Banos plus au nord contiennent une association de microfaune riche en organismes benthiques, que l'on retrouve dans les silex. La détermination des foraminifères provenant des calcaires associés a été publiée par Neumann (1958) et Feinberg (1964). Les auteurs ont cité : Lepidorbitoide socialis, L minor, Clypeorbis mamillata, Hellenocyclina, Siderolites calcitrapoides, S. vidali. (fig. 3 et 4 )

Figure 3 - Échantillon géologique (prélèvement Fernandes 2010), photographies à la loupe binoculaire d'un silex prélevé au sommet des sables fauves à Audignon-Cazaoubidaou (coordonnées géographiques : longitude $=0^{\circ} 39^{\prime} 27^{\prime \prime} 0$, latitude $=43^{\circ} 42^{\prime} 51^{\prime \prime} \mathrm{N}$ ). Clichés P. Fernandes, DAO P.Tallet.

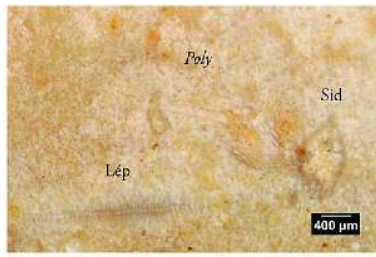

1 : vue générale, texture wackessome, Lépidorbitoids 1 : vue générale, texture wadkestone, Le
Siderolite er Polygonella échelle $400 \mu \mathrm{m}$.

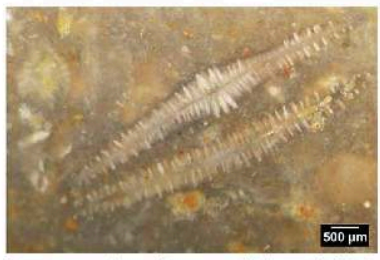

3: texture wnckessone à coupes mériciennes de Lép:3: texture wackestone a coup
dorbiroides, échelle $500 \mu \mathrm{m}$.

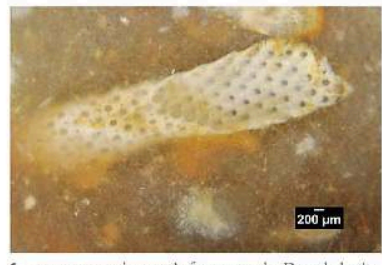

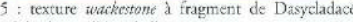
(Cyindropoportlla), b́chelle 200 um
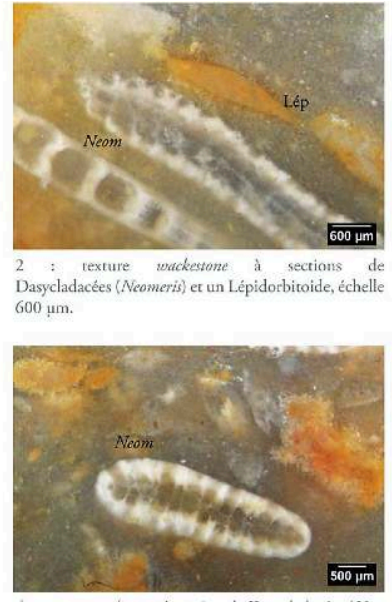

4 : texnure wack 4: texture wackestone 2 is
meris), échelle $500 \mathrm{~mm}$.

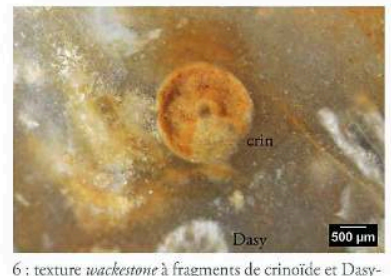

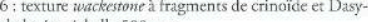
cladacées, échelle $500 \mu m$. 
Figure 4 - Échantillon géologique (prélèvement Fernandes 2010), photographies à la loupe binoculaire d'un silex prélevé dans les altérites au sommet du Maastrichtien à Audignon-Dumes (coordonnées géographiques : longitude $=0^{\circ} 35^{\prime} 34^{\prime \prime} 0$, matitude $=43^{\circ} 42^{\prime} 34,4^{\prime \prime} \mathrm{N}$ ). Clichés : $\mathrm{P}$. Fernandes, DAO : P. Tallet.
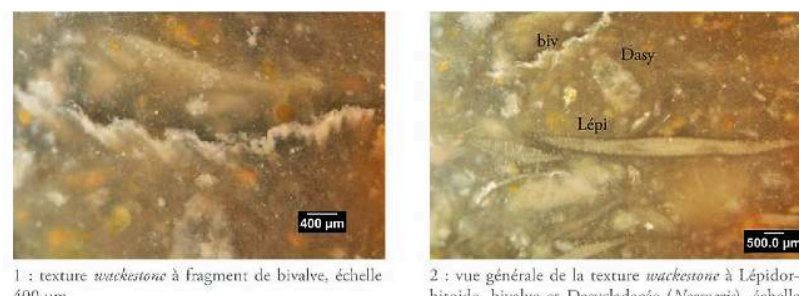

$400 \mu \mathrm{m}$.

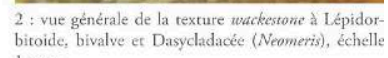
$1 \mathrm{~mm}$.

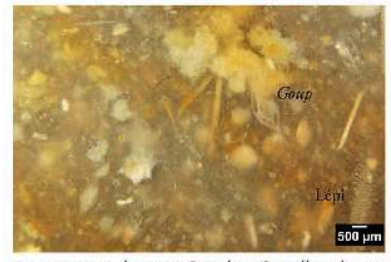
3: texture wackestone à Spiculcs, Gompillatendina et Lépidorbitoides, échelle 500 um.

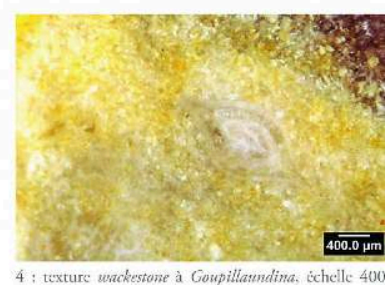

$\mu \mathrm{m}$.
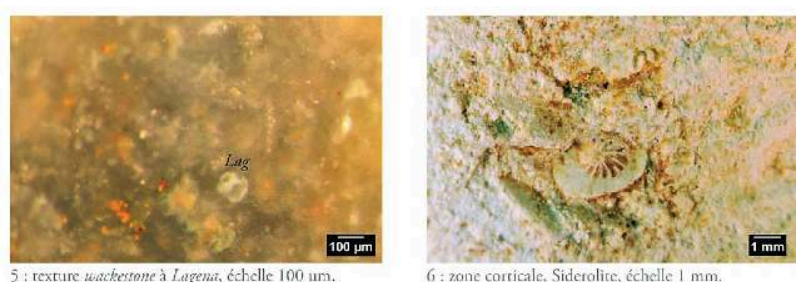

Les rognons de silex sont irréguliers ou réguliers. Ils font $10 \mathrm{à} 20 \mathrm{~cm}$ de longueur, mais certains dépassent $40 \mathrm{~cm}$. En position primaire, le cortex est épais et crayeux. Le silex, gris à l'origine, devient brun assez rapidement lorsqu'il se trouve en position subprimaire (à proximité de la position primaire) et secondaire. La patine est courante car la majorité des échantillons étudiés provient des sables miocènes et notamment des secteurs à cuirasse ferrugineuse. La plupart des gîtes à silex présents sur l'anticlinal d'Audignon sont donc des gîtes sub-primaires et secondaires alimentés par le démantèlement des calcaires campaniens et surtout maastrichtiens.

Les échantillons collectés par nos soins en 2010 correspondent à ces descriptions. La structure est homogène et parfois bioturbée. La texture est surtout wackestone. La matrice est microcristalline à cryptocristalline. Les formes peloïdes sont abondantes. Leur taille oscille entre 10 et $20 \mu \mathrm{m}$. Les intraclastes représentent moins de $10 \%$ de la matrice. Ils sont anguleux et leur taille ne dépasse pas les $200 \mu \mathrm{m}$. Les bioclastes sont abondants, ils représentent $20 \%$ des éléments figurés. La macrofaune comprend des fragments de bivalves, d'ostracodes et d'échinodermes. Les spicules sont plus ou moins bien représentés. Les bryozoaires sont rares ou abondants selon le faciès. Mais la grande différence avec les types déjà décrits, tient au fait que les formes benthiques sont surreprésentées par rapport aux formes planctoniques. Toutes ces remarques concourent à la définition d'un milieu de dépôt de plateforme interne, ouverte tout de même aux influences océaniques (présence des formes planctoniques). On a recensé de très rares pithonelles dans certains échantillons. 


\section{Conclusion et perspectives}

32 Ce travail de cartographie et d'inventaire, à l'échelle du sud de la France, représente le premier volet d'un outil approprié pour l'étude des comportements humains face à la matière première. Nous disposons désormais de données gîtologiques conséquentes et de fiches de caractérisation harmonisées pour plusieurs centaines de types. La compilation de ces notices descriptives devra déboucher sur l'élaboration d'un atlas des microfaciès. Cette démarche collective propose les bases nécessaires au développement d'études interrégionales sur la reconnaissance des stratégies territoriales. La moisson de documents et d'observations, la rigueur des modes de prélèvement et le protocole de diagnose renouvelé aboutissent à des déterminations plus précises du positionnement des matériaux, surtout dans le cas des formations superficielles. A moyen terme, il a été convenu d'intégrer la totalité des données sur les géoressources (appuyées à un SIG) à l'Atlas des patrimoines (http://atlas.patrimoines.culture.fr) pour harmoniser les bases de données, tant au niveau de la cartographie, que des notices explicatives et des fiches de microfaciès.

Notre programme, pour mieux adhérer à la réalité des problèmes que posent les interactions hommes/milieux, doit dépasser le stade de la compilation des données. Nous avons opté, récemment, pour une représentation dynamique, où chaque gîte primaire est présenté en connexion avec l'ensemble des formations superficielles qui sont en lien. Sur cette troisième génération de carte, nous privilégions le type et rassemblons tous les affleurements qui présentent le même faciès lithologique. La carte doit représenter les principaux gites primaires et secondaires à silex, non pas sous forme d'entités distinctes mais connectées. Cette approche, qui permet de suivre chaque type de silex, de visualiser sa dispersion naturelle et de fusionner ces données avec les données archéologiques, conduit à une meilleure définition des lieux de collecte.

\section{BIBLIOGRAPHIE}

AFFOLTER J. 2009 - Les Douattes (Musièges, Haute-Savoie) : les séries magdaléniennes et aziliennes des fouilles Jayet et Pion (1999-2002) in : G. Pion et L. Mevel (dir.) La fin du Paléolithique supérieur dans les Alpes du nord et le jura méridional. Approches culturelles et environnementales. Projet collectif de recherche dirigé par Gilbert Pion. Mémoire de la Société préhistorique française, 50, 161-172 p.

AFFOLTER J. et BRESSY C. 2009 - les matières premières siliceuses : méthodes d'études et ressources In : G. Pion et L. Mevel (dir.) La fin du Paléolithique supérieur dans les Alpes du nord et le jura méridional. Approches culturelles et environnementales. Projet collectif de recherche dirigé par Gilbert Pion. Mémoire de la Société préhistorique française, 50, 143-159 p.

BRESSY C. 2009 - Approvisionnements en silex à l'azilien récent : le site de Gerbaix (SaintChristophe, Savoie) In : G. Pion et L. Mevel (dir.) La fin du Paléolithique supérieur dans les Alpes 
du nord et le jura méridional. Approches culturelles et environnementales. Projet collectif de recherche dirigé par Gilbert Pion. Mémoire de la Société préhistorique française, 50, 173-194 p.

BRESSY C., ANDRÉ P., FERNANDES P., PIBOULE M., REY P.-J. 2007 - Réseau de lithothèques en Rhône-Alpes, rapport d'activités du Projet Collectif de Recherche, 105 p.

DAMOUR A. 1865 - Sur la composition des Haches en pierre trouvées dans les monuments celtiques et chez les sauvages, Comptes Rendus de l'Académie des Sciences, LXI, séances du 21 et 28 août $1865: 1-13$.

FEINBERG H. 1964 - Contribution à l'étude stratigraphique et structurale du dôme d'Audignon, Thèse de 3ème cycle, Université de Paris, $182 \mathrm{p}$.

FOUCAULT J. et RAOULT J.-F. 1997 - Dictionnaire de géologie. Masson, Paris, 4e édition, 324 p.

GUIBERT R. 2000 - Gestion des industries lithiques mésolithiques et néolithiques du Sud-Est de la France,Thèse de 3ème Cycle, Université Paris-I, 369 p.

NEUMANN M. 1958 - Révision des Orbitoïdes du Crétacé et de l'Éocène en Aquitaine occidentale, Thèse de 3ème cycle, Université de Paris, $382 \mathrm{p}$.

OGG J G. 2010 - Latest version (Sept. 2010) of the International Stratigraphic Chart. Copyright ( 2010 International Commission on Stratigraphy chart produced by the ICS.

PRIMAULT J. 2003 - Exploitation et diffusion des silex de la région du Grand-Pressigny au Paléolithique, Thèse de doctorat, Université Paris X, 362 p.

TURQ A. 2005 - Réflexions méthodologiques sur les études de matières premières lithiques. 1 Des lithothèques au matériel archéologique, Paleo 17, p. 111-131.

\section{RÉSUMÉS}

Une carte des principales formations à silex du sud de la France est en cours de réalisation. Elle propose, à tous les préhistoriens, une base nécessaire au développement d'études interrégionales sur la circulation des silex. Elle est le fruit d'une collaboration entre des acteurs impliqués dans la problématique de caractérisation de la provenance des silex. Elle regroupe les résultats de leurs prospections systématiques ou ciblées dans six régions (Aquitaine, Auvergne, LanguedocRoussillon, Midi-Pyrénées, Provence-Alpes-Côte-d'Azur, Rhône-Alpes). Elle intègre, en plus, le dépouillement d'un grand nombre de documents : i) les principaux articles et thèses traitant des formations à silex du sud de la France ; ii) plus de 200 fiches issues de la base de données du soussol BSS du BRGM, qui permettent de visualiser des logs ou des documents scannés ; iii) 529 cartes géologiques à $1 / 50000$ et leurs notices. La carte est organisée en trois couches de données superposables : une carte des affleurements ou gîtes primaires, une carte des altérites et des formations superficielles remaniées et une carte des formations alluviales. La carte existera dans deux versions numériques aisément actualisables: une version dans un format PDF et une version sous la forme d'un SIG. C'est l'ensemble de la formation contenant le ou les même(s) type(s) de silex qui est prise en compte, le terme de formation désignant un terrain possédant des caractères communs et qui constitue un ensemble cartographiable. Chacune des formations recensées fait l'objet d'une notice simplifiée qui décrit l'encaissant et - le ou les - type(s) de silex présent(s). Ces notices descriptives et explicatives contiennent des photos à toutes les échelles (de la formation à l'échelle microscopique). Des références bibliographiques géologiques et archéologiques complèteront chaque notice. La version définitive de ces notices constituera un atlas. Les archéologues et géologues disposeront ainsi de fiches descriptives pour chaque type de 
silex et son encaissant. Elles serviront aux diagnoses analytiques (structures, textures et compositions minéralogiques).

A map of the main flint bearing formations in the South of France is under construction. It will provide an essential basis to develop interregional studies about flint procurements and travels. It results from collaboration between actors involved in topic flint sourcing. It includes results of their systematic surveys and studies in six regions (Aquitaine, Auvergne, Languedoc-Roussillon, Midi-Pyrenees, Provence-Alpes - Côte d'Azur, Rhône-Alpes). Moreover, it includes a large number of associated data: i) The main papers and those dealing with flint bearing formations of southern France, ii) more than 200 records from the under-soil database BSS BRGM, which give access to logs or scanned documents iii) 529 geological maps at 1/50 000 and their leaflets. The map is organized in three superimposed layers : a map of outcrops or primary deposits, a map of surficial weathered formation and a revised map of alluvial formations. The map is available in two versions which are easily updatable : a PDF version and an interactive GIS version. In this document, every formation containing the same type (s) (s) of flint is taken into account and forms a mappable entity. Every listed formation is linked to a text which describes the parent rock and/or the type(s) of flint(s). These records contain descriptive and explanatory pictures at different scale (naked eye to microscopic scale). Geological and archaeological references complement every record. The final version will constitute an atlas. Archaeologists and geologists will find description sheets of every type of flint and bearing rock. They will help for analytical diagnoses (structure, texture and mineralogical composition).

\section{INDEX}

Mots-clés : pétroarchéologie, silex, carte, base de données

Keywords : petrography, chert, map, database

\section{AUTEURS}

\section{PAUL FERNANDES}

Paléotime, 272 rue du lycée Polonais, FR-38250 Villard-de-Lans - paul.fernandes@paleotime Université Bordeaux 1, sciences et technologies, UMR 5199 PACEA, Bât. B18, avenue des Facultés, FR-33405 Talence

\section{JEAN-PAUL RAYNAL}

Université Bordeaux 1, sciences et technologies, UMR 5199 PACEA, Bât. B18, avenue des Facultés, FR-33405 Talence - jp.raynal@pacea.u-bordeaux1.fr

Departement of Human Evolution, Max Planck Institute for Evolutionary Anthropology, Deutscher Platz 6, D-04103 Leipzig, Allemagne,

\section{PASCAL TALLET}

Paléotime, 272 rue du lycée Polonais, FR-38250 Villard-de-Lans - pascal.tallet@paleotime.fr

\section{CHRISTOPHE TUFFERY}

INRAP (DST/SMQ) 7 rue de Madrid, FR-75008 Paris - christophe.tuffery@inrap.fr 


\section{MICHEL PIBOULE}

Université Joseph Fourier Grenoble - piboulemichel@gmail.com

\section{MICHELINE SÉRONIE-VIVIEN}

125 avenue d'Eysines, FR-33110 Le Bouscat : rseronie@club-internet.fr

\section{MARIE-ROGER SÉRONIE-VIVIEN}

125 avenue d'Eysines, FR-33110 Le Bouscat : rseronie@club-internet.fr

\section{ALAIN TURQ}

Musée national de Préhistoire, 1 rue du Musée FR-24620 Les Eyzies-de-Tayac alain.turq@culture.gouv.fr

Université Bordeaux 1, sciences et technologies, UMR 5199 PACEA, Bât. B18, avenue des Facultés, FR-33405 Talence

\section{ANDRÉ MORALA}

Musée national de Préhistoire, 1 rue du Musée FR-24620 Les Eyzies-de-Tayac andre.morala@culture.gouv.fr

Université Bordeaux 1, sciences et technologies, UMR 5199 PACEA, Bât. B18, avenue des Facultés, FR-33405 Talence

\section{JEHANNE AFFOLTER}

UMR 5594 AR-GEO-Lab, Université Neuchâtel, Espace Louis-Agassiz 1 CH-2000 Neuchâtel affolterjs@bluewin.ch

\section{DOMINIQUE MILLET}

13 rue Bordas, FR-33400 Talence - prfmillet@orange.fr

\section{FRANÇOISE MILLET}

13 rue Bordas, FR-33400 Talence - prfmillet@orange.fr

\section{FRÉDERIC BAZILE}

Université de Montpellier 3, UMR 5140 « Archéologie des Sociétés Méditerranéennes », 3 route de Mende, FR-34199, Montpellier - fredericbazile@aol.com

\section{PATRICK SCHMIDT}

Département de Préhistoire UMR 7194, Centre de spectroscopie infrarouge, CP 57, 57 rue Cuvier, FR-75231 Paris Cedex 05, France - patrick.schmidt@gmail.com

\section{PASCAL FOUCHER}

SRA Midi-Pyrénées, Hôtel des Chevaliers de Saint-Jean, 32 rue d'Albade, BP 811, FR-31080 Toulouse cedex 6 - pascal.foucher@culture.gouv.fr

\section{VINCENT DELVIGNE}

Université Bordeaux 1, sciences et technologies, UMR 5199 PACEA, Bât. B18, avenue des Facultés, FR-33405 Talence -v.delvigne@pacea.u-bordeaux1.fr 


\section{JÉRÉMIE LIAGRE}

Service archéologique de la ville de Lyon, 10 rue Neyret, FR-69001 Lyon - jeremie.liagre@mairielyon.fr

\section{STÉPHANE GAILLOT}

Service archéologique de la ville de Lyon, 10 rue Neyret, FR-69001 Lyon -

stephane.gaillot@mairie-lyon.fr

\section{ALEXANDRE MORIN}

Paléotime, 272 rue du lycée Polonais, FR-38250 Villard-de-Lans - alexandre.morin@paleotime.fr

\section{MARIE-HÉLÈNE MONCEL}

Muséum national d'histoire naturelle - CNRS, UMR 7194 CNRS Département de Préhistoire, Institut de Paléontologie humaine, 1, rue René Panhard, FR-75013 Paris - moncel@mnhn.fr

\section{JEAN-FRANÇOIS GARNIER}

Société Préhistorique et Historique de Villeneuve-sur-Lot, BP 104, FR-47303 Villeneuve-sur-Lot

\section{CÉLINE LÉANDRI-BRESSY}

Délégation Régionale à la Recherche et à la Technologie (DRRT) 66, Cours Napoléon, FR-20000

Ajaccio - celine.leandri@recherche.gouv.fr 